\title{
Investigation of ionospheric gradients for GAGAN application
}

\author{
K. Ravi Chandra, V. Satya Srinivas, and A. D. Sarma \\ Research and Training Unit for Navigational Electronics, Osmania University, Hyderabad, India
}

(Received October 29, 2007; Revised July 21, 2008; Accepted September 28, 2008; Online published May 29, 2009)

\begin{abstract}
To cater to the needs of aviation applications, GPS Aided GEO Augmented Navigation (GAGAN) system is being implemented over the Indian region. The most prominent parameter affecting the navigation accuracy of GAGAN is ionospheric delay which is a function of TEC. In the equatorial and low latitude regions such as India, TEC is often quite high with large spatial gradients. Carrier phase data from the GAGAN network of Indian TEC Stations is used for estimating ionospheric gradients in multiple viewing directions. Rate of TEC (ROT) and Rate of TEC Index (ROTI) are calculated to identify the ionospheric gradients. Among the satellite signals arriving in multiple directions, the signals which suffer from severe ionospheric gradients are identified and avoided for improving GAGAN positional accuracy. The outcome of this paper will be helpful for improving GAGAN system performance.
\end{abstract}

Key words: GAGAN, ionospheric gradients, ROT, ROTI.

\section{Introduction}

The Global Positioning System (GPS) is a navigation system which provides continuous positioning and timing information under all weather conditions. As the accuracy of the stand alone GPS is not sufficient for category I (CATI) precision approach, Space Based Augmentation Systems (SBAS) are evolved to cater to the needs of aviation applications. GPS Aided GEO Augmented Navigation (GAGAN) is one such system planned in India. The most prominent error affecting the accuracy of GAGAN is the ionospheric delay. The ionosphere is decorrelated with distance and as well as with time causing anomalies. For reliable communication, navigation and surveillance systems, ionospheric gradients need to be thoroughly investigated for improving the performance. The purpose of the gradients is to identify the satellite signals, which are gradient-free in their direction of propagation. As a result, precise TEC data can be obtained from among the visible satellites in multiple directions. To achieve this objective, initially the satellite signals which do not severely suffer from the gradient effects are identified. Subsequently, among the identified satellite signals the best four are considered for accurate positional information using the Dilution of Precision (DOP) approach (Parkinson et al., 1996). Jean-Marie (2006) referred DOP as the effect of 'geometry of satellites' on measurement accuracy. Gang et al. (2003) have proposed a new method 'Cumulative sum', which quickly and reliably detects small but hazardous gradients for Local Area Augmentation System (LAAS) scenario. Walter et al. (2004) reported the effects of large ionospheric gradients on single frequency airborne smoothing filters for Wide Area Augmentation System (WAAS) and LAAS, and also their mitigation tech-

Copyright (c) The Society of Geomagnetism and Earth, Planetary and Space Sciences (SGEPSS); The Seismological Society of Japan; The Volcanological Society of Japan; The Geodetic Society of Japan; The Japanese Society for Planetary Sciences; TERRAPUB. niques. Warnant and Pottiaux (2000) also made an attempt to identify possible correlations between the ionospheric activity and unidentified problems in GPS data processing by duly detecting the travelling ionospheric disturbances. In this paper the estimated ROT and ROTI $(\mathrm{TECu} / \mathrm{min})$ of all visible satellites are used to identify signals which suffer from severe ionospheric gradients.

\section{Results and Discussion}

In the GAGAN network 20 TEC stations are installed to monitor the ionospheric behavior. The data from one of these TEC stations (Lucknow, Lat. $26.76^{\circ} \mathrm{N}$, Long. $80.88^{\circ} \mathrm{E}$ ) is used for this analysis. The processed GPS data contains 23 parameters. Out of these only seven parameters, namely user position, PRN number of satellite, GPS week number, GPS seconds of the week, elevation angle, azimuth angle and TEC are used in the analysis.

Several days of data corresponding to both quiet and disturbed days are analyzed. However, in this paper the data from various visible Satellite Vehicles (SVs) corresponding to a typical disturbed $\left(7<K_{\mathrm{P}}<8\right)$ day (25th July, 2004) is processed and analyzed for identifying the ionospheric gradients. The sampling rate of data is 1 minute.

The rate of change of the ionospheric delay (ROT) due to satellite signals received at the receiver is given as (Warnant and Pottiaux, 2000)

$$
\operatorname{ROT}_{u}^{k}\left(t_{i}\right)=\frac{\operatorname{TEC}_{u}^{k}\left(t_{i}\right)-\mathrm{TEC}_{u}^{k}\left(t_{i-1}\right)}{t_{i}-t_{i-1}}(\mathrm{TECu} / \mathrm{min})
$$

Where $\operatorname{ROT}_{u}^{k}\left(t_{i}\right)=$ Rate of TEC estimated at time epoch $t_{i}$ by receiver $(u)$ on satellite $(k), \operatorname{TEC}_{u}^{k}\left(t_{i}\right)$ and $\operatorname{TEC}_{u}^{k}\left(t_{i-1}\right)=$ Total Electron Content measured by receiver $(u)$ on satellite $(k)$ at epoch $t_{i}$ and epoch $t_{i-1}$ respectively.

ROT is a function of $K_{\mathrm{p}}$, viewing direction and magnetic activity. Secondly, these ROT values are converted from slant to vertical using a standard mapping function $M(E)$ 


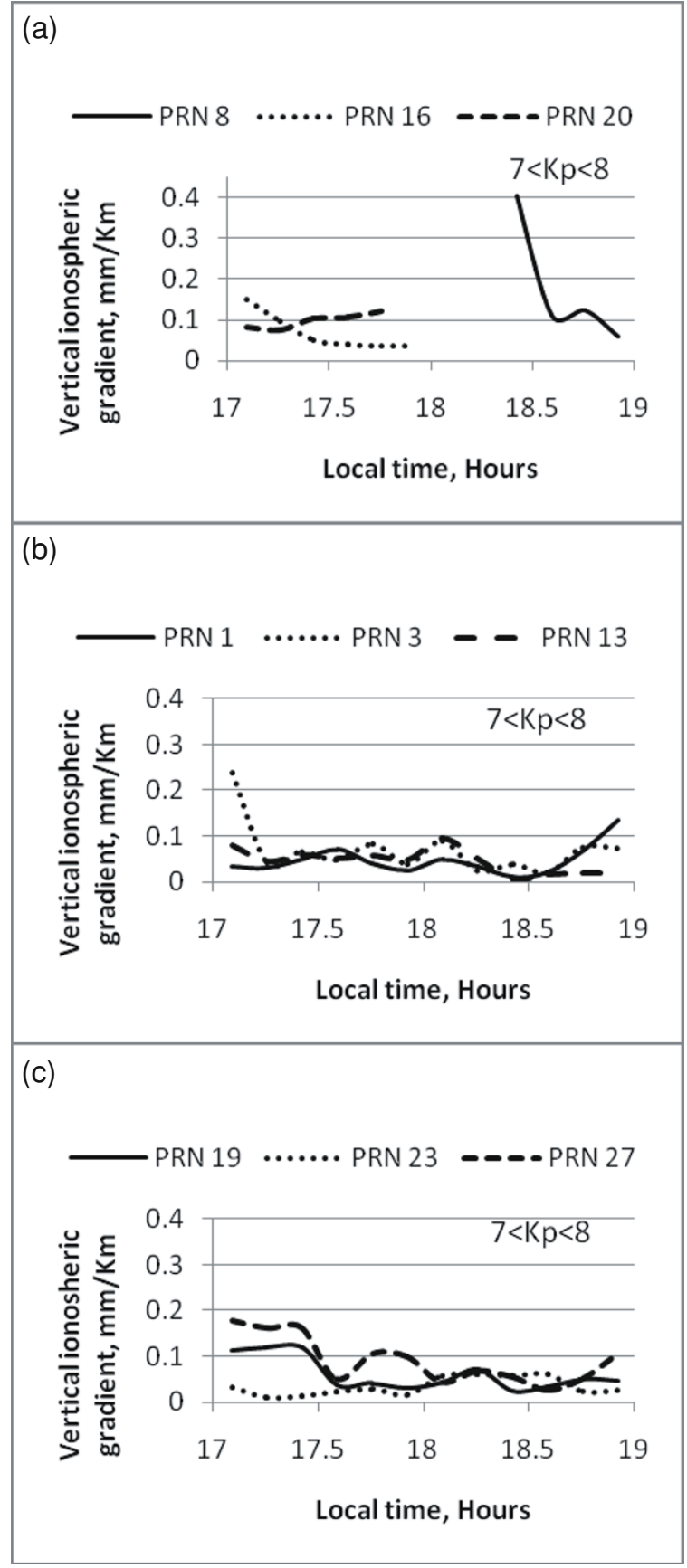

Fig. 1. Variation of vertical ionospheric gradient for all visible multiple viewing satellites based on Lucknow GAGAN network TEC stations (25th July, 2004) over a local time period (17:00-19:00 Hrs).

(Guochang, 2003)

$$
M(E)=\left\{1-\left(\frac{R_{\mathrm{E}}}{R_{\mathrm{E}}+h}\right)^{2} \cos ^{2} E\right\}^{1 / 2} .
$$

Where $E=$ Elevation angle at the reference station (in degrees),

$h=$ Altitude of the ionospheric shell above the surface of the Earth $(350 \mathrm{Km})$

$R_{\mathrm{E}}=$ Earth radius $(6378 \mathrm{Km})$.

The slant ROT is averaged over 10 minutes to obtain

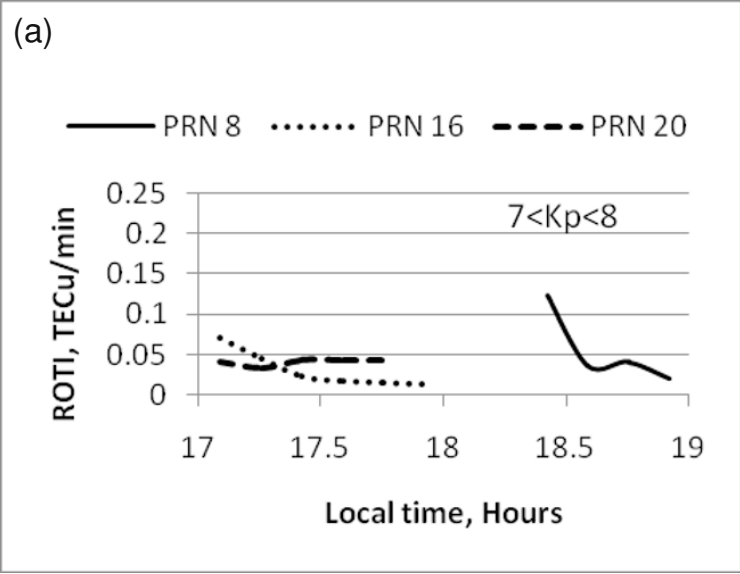

(b)

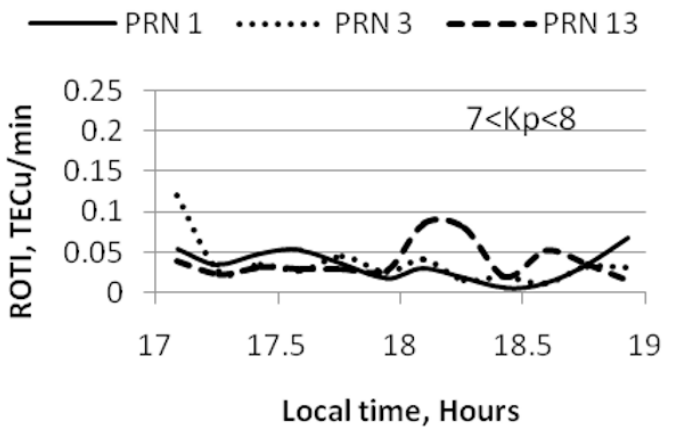

(c)
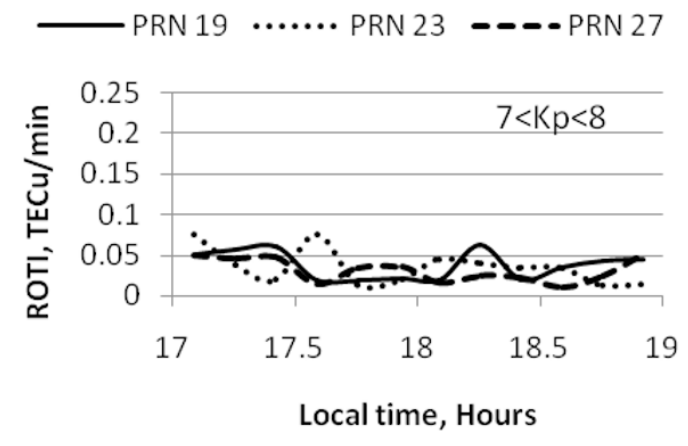

Fig. 2. Variation of ROTI for all visible multiple viewing satellites based on Lucknow GAGAN network TEC stations (25th July, 2004) over a local time period (17:00-19:00 Hrs).

$\langle$ ROT $\rangle$. ROTI is standard deviation of ROT and is given as (Krankowski et al., 2005)

$$
\mathrm{ROTI}=\sqrt{\left\langle\mathrm{ROT}^{2}\right\rangle-\langle\mathrm{ROT}\rangle^{2}}(\mathrm{TECu} / \mathrm{min})
$$

ROTI measurements can be used to predict the presence of ionospheric scintillation also (Basu et al., 1999). Its values signify the presence of only temporal gradients. In order to identify spatial gradients, Jiyun et al. (2006) proposed a 'Time step method'. By considering the distance between the Ionospheric Pierce Point (IPP) at epoch $t_{i}$ and IPP at epoch $t_{i-1}$, the vertical ionospheric gradient $\left(\sigma_{\mathrm{VIG}}\right)$ is esti- 
mated as

$$
\sigma_{u \mathrm{VIG}}^{k}\left(t_{i}\right)=\frac{\operatorname{TEC}_{u}^{k}\left(t_{i}\right)-\mathrm{TEC}_{u}^{k}\left(t_{i-1}\right)}{D_{t_{i}, t_{i-1}}}(\mathrm{~mm} / \mathrm{Km}) .
$$

Where $D_{t_{i}, t_{i-1}}=$ Distance between the IPPs corresponding to time epochs $t_{i}$ and $t_{i-1}$.

As the delays due to different epochs were considered and divided by the corresponding separation, the estimated $\sigma_{\mathrm{VIG}}$ is a combination of both temporal and spatial gradients.

The typical data from various visible satellites corresponding to two hours (Local time: 17:00-19:00 Hrs) are processed and analyzed. Variations of $\sigma_{\mathrm{VIG}}$ with respect to local time are presented for selected visible satellites (Fig. 1). At $17.08 \mathrm{Hrs}$, eight satellites are visible. After about $17.74 \mathrm{Hrs}$, SV 20 and after 17.91 Hrs, SV 16 have gone out of sight and only six satellites are visible. After about 18.24 hours, the SV 8 has come in to line of sight to reference station. Hence, there exists a corresponding trace of ROTI curve after 18.24 hour onwards. From the data, it is evident that, at any point of time GPS receiver is in line of sight with a minimum six and a maximum eight satellites. To investigate the effect due to temporal gradients, variation of ROTI with respect to local time is presented for all visible satellites (Fig. 2). It is shown that for the entire time period ROTI and $\sigma_{\mathrm{VIG}}$ for SVs 1, 13, 16, 19, 20, 23 and 27 has not exceeded the maximum values of $0.085 \mathrm{TECu} / \mathrm{min}$ (PRN 16) and $0.177 \mathrm{~mm} / \mathrm{Km}$ (PRN 27) respectively. Further, there are no abrupt changes in the plots. It signifies that impact of ionospheric anomalous activity $\left(7<K_{\mathrm{p}}<8\right)$ on different viewing SVs has the similar effect. Except for a few epochs, SVs 3 and 8 also show similar trend. At local time of $18.41 \mathrm{Hrs}$, both ROTI $(0.1238 \mathrm{TECu} / \mathrm{min})$ and $\sigma_{\mathrm{VIG}}$ $(0.4047 \mathrm{~mm} / \mathrm{Km})$ are identified to be the maximum due to PRN 8. From both Figs. 1 and 2, it is observed that, the plots exhibit similar trend. It signifies that even though $\sigma_{\mathrm{VIG}}$ is a combination of both temporal and spatial gradients, the role of temporal gradients seem to be predominant. The reason for this could be less spatial variation between the successive IPPs. This is in turn based upon the radial movement of satellite (angular velocity of approx. $3.9 \mathrm{Km} / \mathrm{sec}$ ) and user.

On estimation of gradients an attempt is made to improve the accuracy and integrity of user position. As example estimation, a time epoch of $17.08 \mathrm{Hrs}$ is considered. Out of all the visible satellites, $\sigma_{\mathrm{VIG}}$ values of SV $3(0.2378 \mathrm{~mm} / \mathrm{Km})$ and SV $27(0.1777 \mathrm{~mm} / \mathrm{Km})$ are found to be relatively higher than the rest of the SVs. Therefore, the signals from these two SVs are avoided. Consequently, among the available SVs, only six will remain. Among these six SVs, the best four are chosen, based on DOP geometry for accurate position estimation. Since there are six visible satellites DOP geometry is to be checked only for 15 combinations of four satellites. In this way we are able to avoid the signals affected by ionospheric gradients and also minimize the DOP computations from 70 to 15 for selection of the best $4 \mathrm{SVs}$.

\section{Conclusions}

In order to provide accurate position as well as integrity information, investigation of ionospheric gradients is necessary for GAGAN applications. The outcome of this work can be extended for the LAAS system for improving performance of Required Navigation Performance (RNP) parameters viz. accuracy, availability, integrity and continuity to achieve CAT II accuracy requirement of civil aviation applications. This paper emphasizes that, precise estimation and identification of ionospheric gradients over a low latitude region is necessary to achieve better accuracies. This will help to identify relatively gradient-free propagation paths, among the multiple viewing directions of satellites. Subsequently, among the available satellite signals the best four can be chosen to achieve best DOP value for improving positional and integrity information.

Acknowledgments. The research work presented in this paper has been carried out under the project entitled "Analysis and modeling of Indian Ionospheric Electron Content for GPS Applications" funded by ISRO, Bangalore, Vide Order No. CAWSES:05, Dated: 28th December, 2005.

\section{References}

Basu, S., K. M. Groves, J. M. Quinn, and P. Doherty, A comparison of TEC fluctuation and scintillation at Ascension Island, J. Atmos. Sol. Terr. Phys., 61, 1219-1226, 1999.

Gang, X., S. Pullen, M. Luo, and P. Enge, Detecting Ionospheric Gradients with Cumulative Sum (CUSUM) Method, American Institute of Aeronautics and Astronautics, Inc. (AIAA 2003-2415), 2003.

Guochang, Xu., GPS Theory, Algorithms and Applications, SpringerVerlog, Berlin, Germany, 2003.

Jean-Marie, Z., Essentials of Satellite Navigation, Ublox compendium, GPS-X-02007-C, 2006.

Jiyun, L., S. Pullen, S. Datta-Barua, and P. Enge, Assessment of Nominal Ionosphere Spatial Decorrelation for LAAS, IEEE/ION PLANS 2006, pp. 506-514, 2006.

Krankowski, A., I. I. Shagimuratov, Lubomir, W. Baran, and I. I. EphishoV, Study of TEC fluctuations in Antarctic Ionosphere during storm using GPS observations, Acta Geophysic Apolonica, 53(2), 205-218, 2005.

Parkinson, B. W., GPS Theory and Applications, pp. 81-114, American Institute of Aeronautics and Astronautics, Inc, Volume I, Washington, 1996.

Walter, T., S. Datta-Barua, J. Blanch, and P. Enge, The Effects of Large Ionospheric Gradients on Single Frequency Airborne Smoothing Filters for WAAS and LAAS, Institute of Navigation's National Technical Meeting, San Diego CA, January 2004.

Warnant, R. and E. Pottiaux, The increase of the ionospheric activity as measured by GPS, Earth Planets Space, 52, 1055-1060, 2000.

K. Ravi Chandra, V. Satya Srinivas, and A. D. Sarma (e-mail: ad_sarma@yahoo.com) 